\title{
Long-term Monitoring of Molonglo Calibrators
}

\author{
B. M. Gaensler ${ }^{1,2,3}$ and R. W. Hunstead ${ }^{2}$ \\ ${ }^{1}$ Center for Space Research, Massachusetts Institute of Technology, 70 Vassar Street, \\ Cambridge, MA 02139, USA \\ bmg@space.mit.edu \\ ${ }^{2}$ School of Physics, University of Sydney, NSW 2006, Australia \\ rwh@physics.usyd.edu.au \\ ${ }^{3}$ Hubble Fellow \\ Received 1999 August 20, accepted 2000 January 9
}

\begin{abstract}
Before and after every 12 hour synthesis observation, the Molonglo Observatory Synthesis Telescope (MOST) measures the flux densities of $\sim 5$ compact extragalactic radio sources, chosen from a list of 55 calibrators. From 1984 to 1996, the MOST made some 58,000 such measurements. We have developed an algorithm to process this dataset to produce a light curve for each source spanning this thirteen-year period. We find that 18 of the 55 calibrators are variable, on time scales between one and ten years. There is the tendency for sources closer to the Galactic Plane to be more likely to vary, which suggests that the variability is a result of refractive scintillation in the Galactic interstellar medium. The sources with the flattest radio spectra show the highest levels of variability, an effect possibly resulting from differing orientations of the radio axes to the line of sight.
\end{abstract}

Keywords: ISM: general—quasars: general—radio continuum: galaxies

\section{Introduction}

Many compact extragalactic radio sources show variations in their radio flux density as a function of time. At high frequencies $(\nu \gg 1 \mathrm{GHz})$ this variability is usually interpreted as being intrinsic to the source (e.g. Qian et al. 1995, although see Kedziora-Chudczer et al. 1997). Variability at lower frequencies (e.g. Hunstead 1972; Ghosh \& Rao 1992) is normally attributed to refractive interstellar scintillation, in which the intensity variations are caused by distortions of the wavefront by electron density gradients in an intervening screen of material (Shapirovskaya 1978; Rickett, Coles \& Bourgois 1984). There is evidence that the parameters of such variability depend on the Galactic latitude of the source (Spangler et al. 1989; Ghosh \& Rao 1992), suggesting that the material causing the scintillation is in our own Galaxy.

In some sources, both intrinsic variability and scintillation may be occurring at the same time (e.g. Mitchell et al. 1994). Such sources show large but uncorrelated variations at high and low frequencies. At frequencies $\nu \approx 1 \mathrm{GHz}$, one might expect both effects to occur; however, variability in this region of the spectrum is largely unexplored. The Molonglo Observatory Synthesis Telescope (MOST; Mills 1981; Robertson 1991) operates at a frequency of $843 \mathrm{MHz}$, and is thus well placed to study this regime. For calibration purposes, the MOST monitors the flux density of $\sim 10$ compact extragalactic sources every

(C) Astronomical Society of Australia 2000 day. Thus the full record of MOST calibrations, running from 1984 until the commencement of the Wide Field Project in 1996 (Large et al. 1994), forms an ideal database with which to study variability in this intermediate frequency range.

A preliminary analysis of three MOST calibrators was made by Campbell-Wilson \& Hunstead (1994, hereafter Paper I). It was shown that flux density measurements with a relative accuracy of $2 \%$ could be extracted from the database. Over the period from 1990.1 to 1993.7, the source MRC B0409-752 was shown to be stable, while MRC B0537-441 and MRC B1921-293 were found to be highly variable. In this paper we now report on all 55 calibrators used by the MOST, over a thirteen-year period. In Section 2 we explain how we process the calibrator measurements in order to produce light curves for each source, and then determine whether a source is variable or not. In Section 3 we present light curves for all 55 sources, plus structure functions for those sources found to be variable. In Section 4 we discuss some individual sources in our sample, and consider whether any of the observed properties correlate with Galactic latitude.

\section{Observations and Data Analysis}

\subsection{SCAN Measurements}

The MOST is an east-west synthesis telescope, consisting of two cylindrical paraboloids of dimensions $778 \mathrm{~m} \times 12 \mathrm{~m}$. Radio waves are received by a 
line feed system of 7744 circular dipoles. The telescope is steered by mechanical rotation of the cylindrical paraboloids about their long axis, and by phasing the feed elements along the arms. In a single 12-hour synthesis, the MOST can produce an image at a spatial resolution of $43^{\prime \prime} \times 43^{\prime \prime} \operatorname{cosec}(|\delta|)$ and at a sensitivity of $\sim 1$ mJy beam $^{-1}$ (where 1 jansky $[\mathrm{Jy}]=10^{-26} \mathrm{~W} \mathrm{~m}^{-2} \mathrm{~Hz}^{-1}$ ).

Before and after each 12-hour synthesis, the MOST typically observes $\sim 5$ calibration sources in fan-beam "SCAN" mode in order to determine the gain and pointing corrections for the telescope. These sources are chosen from a list of 55 calibrators, 45 of which were chosen from the Molonglo Reference Catalogue (MRC) at $408 \mathrm{MHz}$ (Large et al. 1981), using as selection criteria that they have declination $\delta<-30^{\circ}$, Galactic latitude $|b|>10^{\circ}$, angular sizes $<10^{\prime \prime}$ and flux densities $S_{408 \mathrm{MHz}}>4 \mathrm{Jy}$ and $S_{843 \mathrm{MHz}}>2.5 \mathrm{Jy}$; further discussion is given by Hunstead (1991). This list was later supplemented by seven flat-spectrum $\left(S_{408 \mathrm{MHz}}<4 \mathrm{Jy}\right)$ sources from the work of Tzioumis (1987), plus three compact sources for which $\delta>-30^{\circ}$. The full list of calibrators is given in Paper I.

For each SCAN observation the calibrator source is tracked for two minutes, after which the mean antenna response is compared with the theoretical fan-beam response to a point source. From 1994 to 1996, over 58,000 such measurements were made. In each case, parameters such as the goodnessof-fit of the response and the pointing offset from the calibrator position are recorded, along with an amplitude which is the product of the instantaneous values of the source flux density, the intrinsic telescope gain and local sensitivity factors. The main factors are strong but welldetermined functions of meridian distance ${ }^{1}(\mathrm{MD})$ and of ambient temperature (which ranges from $-10^{\circ} \mathrm{C}$ to $+40^{\circ} \mathrm{C}$ during the year); the variation of sensitivity with MD is shown in Figure 1. After applying corrections for these two factors, the telescope gain for each SCAN is derived by comparing the corrected amplitude with the tabulated flux density of the corresponding source (see Table 1 of Paper I). The residual scatter in the gain determined from steady sources (defined in Section 2.3) is typically $2 \%$ RMS; this is the fundamental limit to the uncertainty of measurements made using the SCAN database.

\subsection{Selection Criteria}

Various selection criteria are applied to the SCAN database before accepting measurements for further analysis:

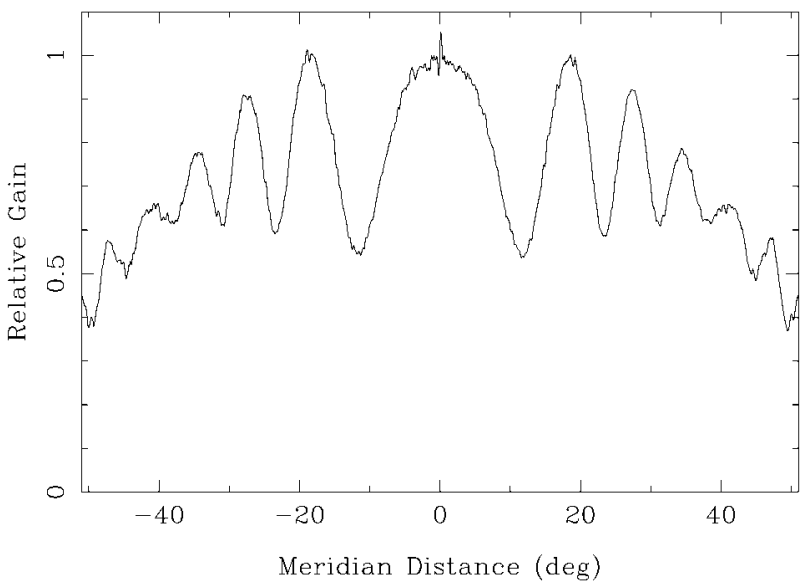

Figure 1-The relative gain of the MOST as a function of meridian distance. The data shown correspond to the asymmetric gain curve of Burgess (personal communication), with additional empirical corrections of order $2 \%$.

- The uncertainties in the MD gain curve increase towards large MD, and observations made outside the $\mathrm{MD}$ range $\pm 50^{\circ}$ are excluded

- Observations made during routine performance testing (characterised by a large number of successive SCANs of the same source) are discounted, except where the standard deviation in gain was less than $5 \%$. In such cases the group is treated as a single measurement with a gain equal to the average of the group

- A poor fit to the antenna response can often indicate a confusing source or a telescope malfunction, and such data are excluded

- Extreme values of the relative gain (below 0.5 or above 1.5 ) are assumed to be discrepant and are discarded.

Because calibration observations are made just before and after each synthesis, the database is typically clustered into SCANs closely spaced in time. We define a 'block' as a group of at least three valid observations made within the space of an hour. We initially exclude observations of 15 of the 55 calibrators (see Table 1 of Paper I) because of: (i) a flat spectrum $\left(\alpha>-0 \cdot 5, S \propto \nu^{\alpha}\right)$, (ii) suspected variability or (iii) the presence of a confusing source. By averaging the gains determined from each SCAN within a block, a representative gain for the telescope at that particular epoch can be determined. This is then applied to each individual observation within the block to obtain a measurement of flux density for that source.

Some of the resultant light curves have thousands of data points, generally sampled at highly irregular intervals. Some light curves have significant scatter; it is not clear whether this scatter is due to

\footnotetext{
${ }^{1}$ Meridian distance, MD, is related to hour-angle, $H$, by $\sin \mathrm{MD} \approx \cos \delta \sin H-$ see Robertson (1991).
} 
unrecognised systematic errors in our flux density determination, to true variability on time-scales shorter than the typical sampling interval, or to the presence of confusing sources in the field. In any case, we chose to bin each light-curve at 30 day intervals; the mean of all flux densities within a given bin becomes a single point on a smoothed light curve, and the standard deviation of the measurements in that bin becomes the error bar associated with this measurement. ${ }^{2}$ While binning the data filters out any genuine variability on time-scales less than a month, the irregular sampling intervals of the observations and the inherent uncertainty in a single SCAN's flux density make the MOST database less than ideal for studying such short-term behaviour.

\subsection{Analysis of Variability}

In order to quantify which sources are variable and which are steady, we calculate the $\chi^{2}$ probability that the flux has remained constant for a given source (e.g. Kesteven, Bridle \& Brandie 1976). We first calculate the quantity

$$
x^{2}=\sum_{i=1}^{n}\left(S_{i}-\tilde{S}\right)^{2} / \sigma_{i}^{2},
$$

where $\tilde{S}$ is the weighted mean, given by

$$
\tilde{S}=\sum_{i=1}^{n}\left(S_{i} / \sigma_{i}^{2}\right) / \sum_{i=1}^{n}\left(1 / \sigma_{i}^{2}\right),
$$

and where $S_{i}$ is the $i$ th measurement of the flux density for a particular source, $\sigma_{i}^{2}$ is the variance associated with each 30-day estimate of $S_{i}$, and $n$ is the number of binned data points for that source. For normally-distributed random errors, we expect $x^{2}$ to be distributed as $\chi^{2}$ with $n-1$ degrees of freedom. For each source, we can then calculate the probability $P$ of exceeding $x^{2}$ by chance for a random distribution.

A high value of $P$ indicates that a source has a steady flux density over the available time period; we classify a source as steady (S) if $P>0 \cdot 01$, and undetermined (U) if $0 \cdot 001<P<0 \cdot 01$. However, the $\chi^{2}$ test cannot distinguish between sources which are genuinely variable and those which simply have a large scatter in their light curve; both light curves result in a low value of $P$. We distinguish between these possibilities by computing the structure function (e.g. Hughes, Aller \& Aller 1992; Kaspi \& Stinebring 1992) for each source for which $P<0 \cdot 001$. The mean is subtracted from the binned time series $S_{t}$, and these data are then normalised by dividing by the standard deviation.
This yields a new time series $F_{t}$, from which the structure function

$$
\Sigma_{\tau}=\left\langle\left[F_{t+\tau}-F_{t}\right]^{2}\right\rangle
$$

can be calculated, where $\tau$ is a parameter known as the lag. If a light curve contains scatter but no true variability, then the structure function will have the value $\Sigma_{\tau} \approx 2$ for all values of $\tau$. But when a source is truly varying, we expect the resulting structure function to consist of three regimes:

- Noise regime: at small lags, $\Sigma_{\tau}$ is more or less constant

- Structure regime: as $\tau$ increases, $\Sigma_{\tau}$ increases linearly (on a log-log plot)

- Saturation regime: at high lags, the structure function turns over and oscillates around $\Sigma_{\tau}=2$ (for our normalisation). If there is a second, longer, time-scale in the data, the structure function can enter another linear regime at longer lags before again saturating.

If a source has $P<0 \cdot 001$ but shows no clear structure in its structure function, we classify it as undetermined (U). Only sources which have both $P<0.001$ and show structure are classified as variable $(\mathrm{V})$. In these cases, the structure function can also be used to obtain a characteristic time scale $\tau_{V}$ for variability; we define $\tau_{V}$ to be equal to twice the lag at which the structure function saturates. We expect a structure function to be sensitive only to time scales longer than about 100 days (i.e. a few multiples of the sampling interval of the binned data). Furthermore, caution should be applied when interpreting structure at large values of $\tau$, as only a few points make a contribution to $\Sigma_{\tau}$ at these long lags (e.g. Hughes et al. 1992).

\section{Results}

Approximately 28000 SCANs meet the selection criteria described in Section 2.2, and around 22000 of these fall within valid blocks. The resulting light curves for the 55 MOST calibrators are given in Figure 2. ${ }^{3}$ Using the criteria described in Section $2.3,18$ sources are found to be variable, 19 are found to have steady light curves, and the remaining 18 are undetermined. Each source in Figure 2 is marked with a $\mathrm{V}, \mathrm{S}$ or $\mathrm{U}$ corresponding to its classification.

Structure functions for the 18 variable sources are shown in Figure 3; for each source we have estimated the time scale for variability, $\tau_{V}$, as marked on each plot. However, we note that some of these estimates are very approximate, as a result of the sparse and/or irregular sampling of the light curves. For example, for MRC B2326-477 we have assigned $\tau_{V}=400 \mathrm{~d}$, but one could just as easily argue that $\tau_{V}=2000 \mathrm{~d}$. Furthermore, there is evidence that

\footnotetext{
${ }^{2}$ In cases where there is only one measurement in a particular 30 day interval, the error is nominally assigned to be $5 \%$ of the measured flux density.

3 The corresponding data tables are available at http://www.physics.usyd.edu.au/astrop/scan/ .
} 


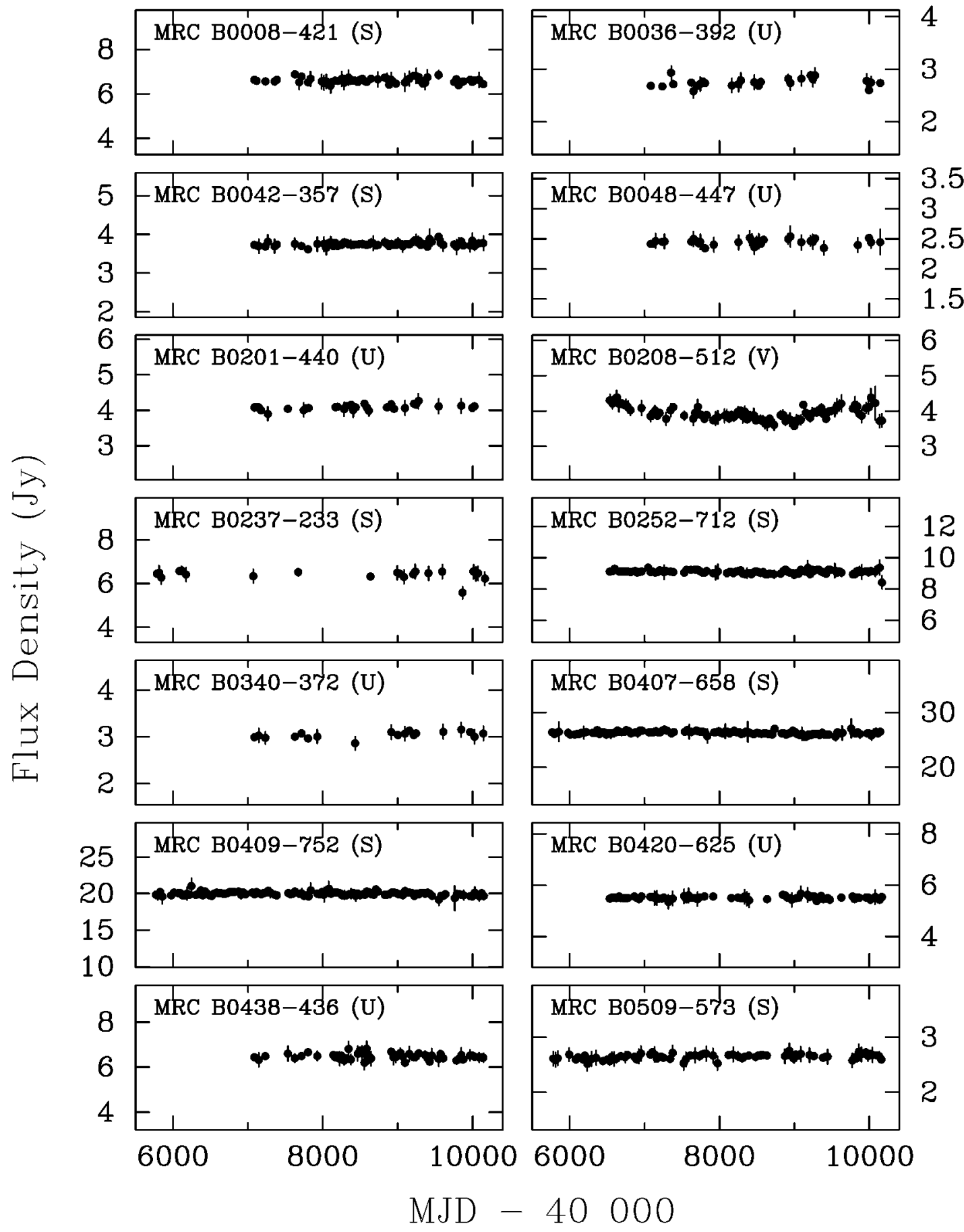

Figure 2-Light curves for 55 MOST calibrators. Sources are marked S, U or V, corresponding to whether their time behaviour is steady, undetermined or variable respectively. Each abcissa ranges between MJD - $40000=5500$ (1983 Jun) and 10400 (1996 Nov), while ordinates run between 0.5 and 1.5 times the nominal flux density for each source (see Table 1 of Paper I). Data have been binned into 30 day intervals - the error bars shown are the $1 \sigma$ standard deviation of the individual data points within each interval, or are set at $5 \%$ in cases where only one data point falls in a given 30 day period.

the structure functions for some sources, such as MRC B1740-517, enter another linear regime beyond the point where they saturate. This suggests that there are variations on time scales longer than we can measure with these data. Some properties of the 18 variable sources are summarised in Table 1.
4 Discussion

\subsection{Individual Sources}

We restrict our comments here to the 18 sources found to be variable. Many of these sources have been observed in snapshot mode at $5 \mathrm{GHz}$ with 


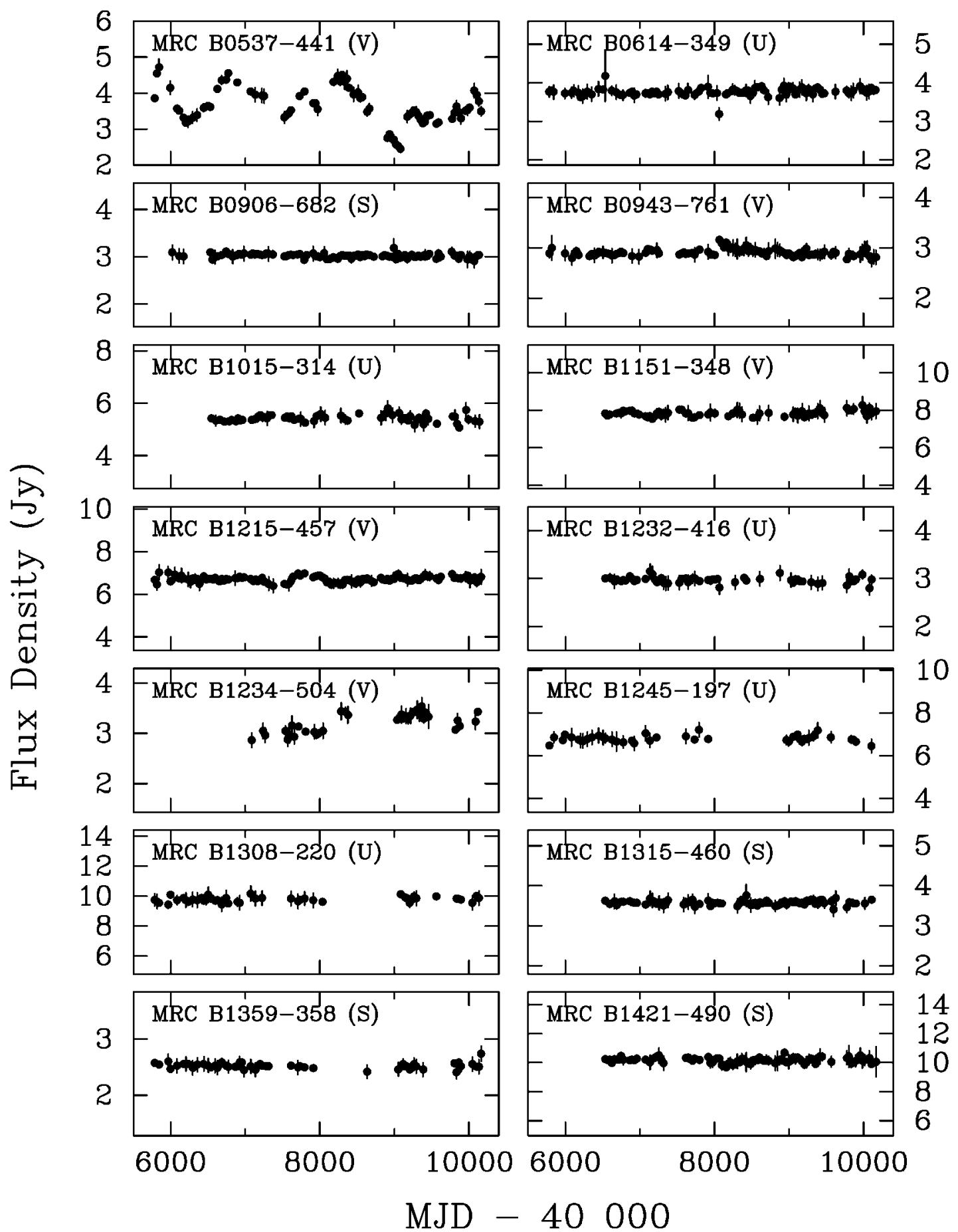

Figure 2-(continued)

the Australia Telescope Compact Array (ATCA, Burgess 1998), and are also ATCA secondary phase calibrators.

MRC B0208-512: VLBI modelling shows a strong core (Preston et al. 1989), and a jet-like feature (Tingay et al. 1996). Detected as an X-ray source in the ROSAT All-Sky Survey (Brinkmann, Siebert \& Boller 1994) and as a $\gamma$-ray source in the EGRET survey (Bertsch et al. 1993).

MRC B0537-441: See Paper I.
MRC B0943-761: Close 2.! double at $5 \mathrm{GHz}$ (Burgess 1998). Detected as an X-ray source in the ROSAT All-Sky Survey (Brinkmann, Siebert \& Boller 1994).

MRC B1151-348: Radio spectrum peaks at $\sim 200$ MHz. A VLBI image shows a 90 mas double structure (King et al. 1993).

MRC B1215-457: Compact steep-spectrum source with a strong, slightly resolved VLBI core (Preston et al. 1989). 


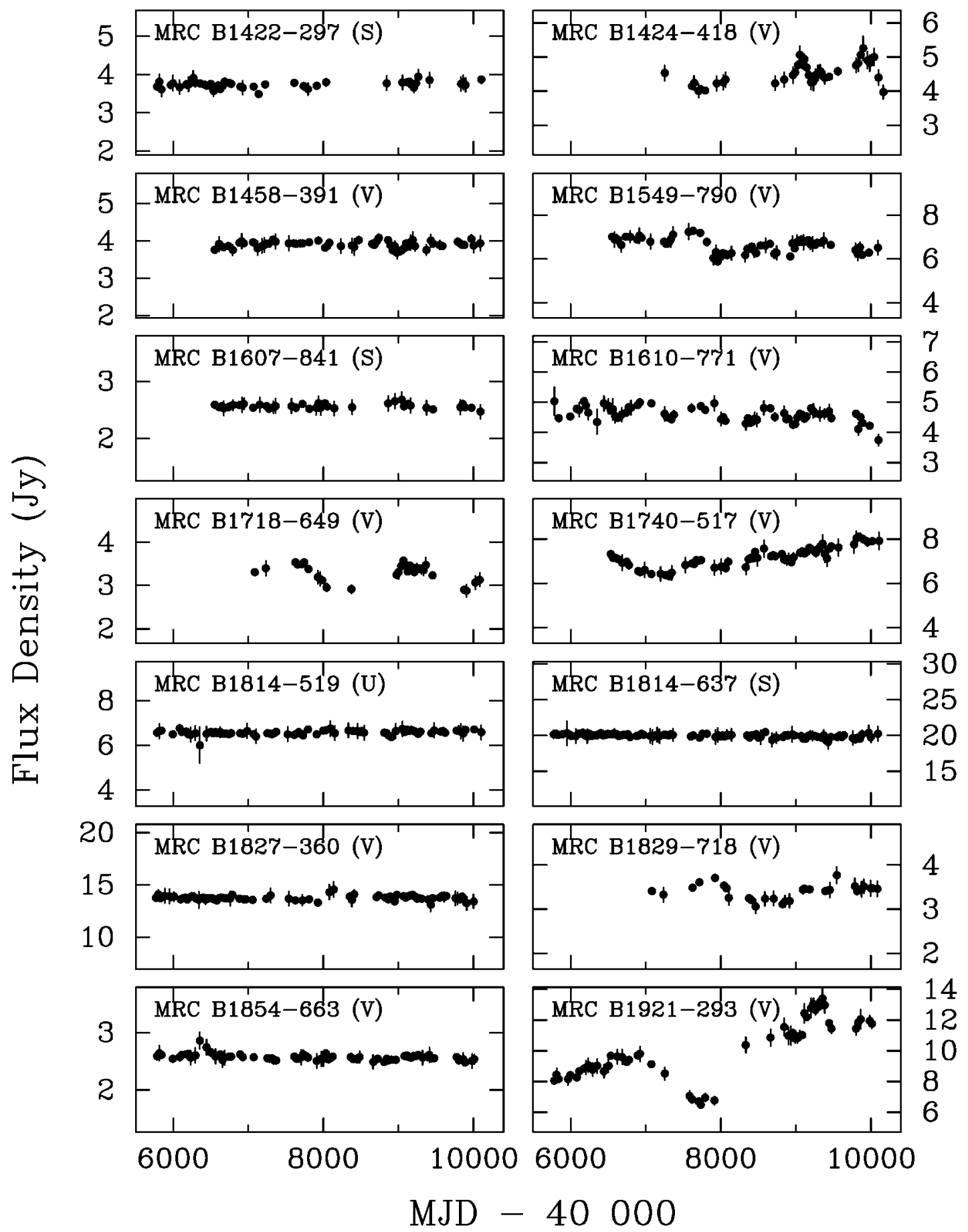

Figure 2-(continued)

MRC B1234-504: Compact steep-spectrum source, with no optical counterpart on the UK Schmidt sky survey but possible stellar identification on a CCD image obtained at the Anglo-Australian Telescope (AAT) (Burgess 1998).

MRC B1424-418: Discordant flux densities measured at Parkes point to the source being variable at $5 \mathrm{GHz}$ (Burgess, personal communication). VLBI modelling shows an unequal 23 mas double structure (Preston et al. 1989).
MRC B1458-391: Compact steep-spectrum source in a crowded optical field; optical ID based on an AAT CCD image (Burgess 1998).

MRC B1549-790: VLBI image shows a curved structure, possibly a core plus jet (Murphy et al. 1993).

MRC B1610-771: Quasar with a flat radio spectrum and very steep optical spectrum (Hunstead \& Murdoch 1980). VLBI observations (Preston et al. 1989) show a strong core surrounded by a 50 mas halo. 


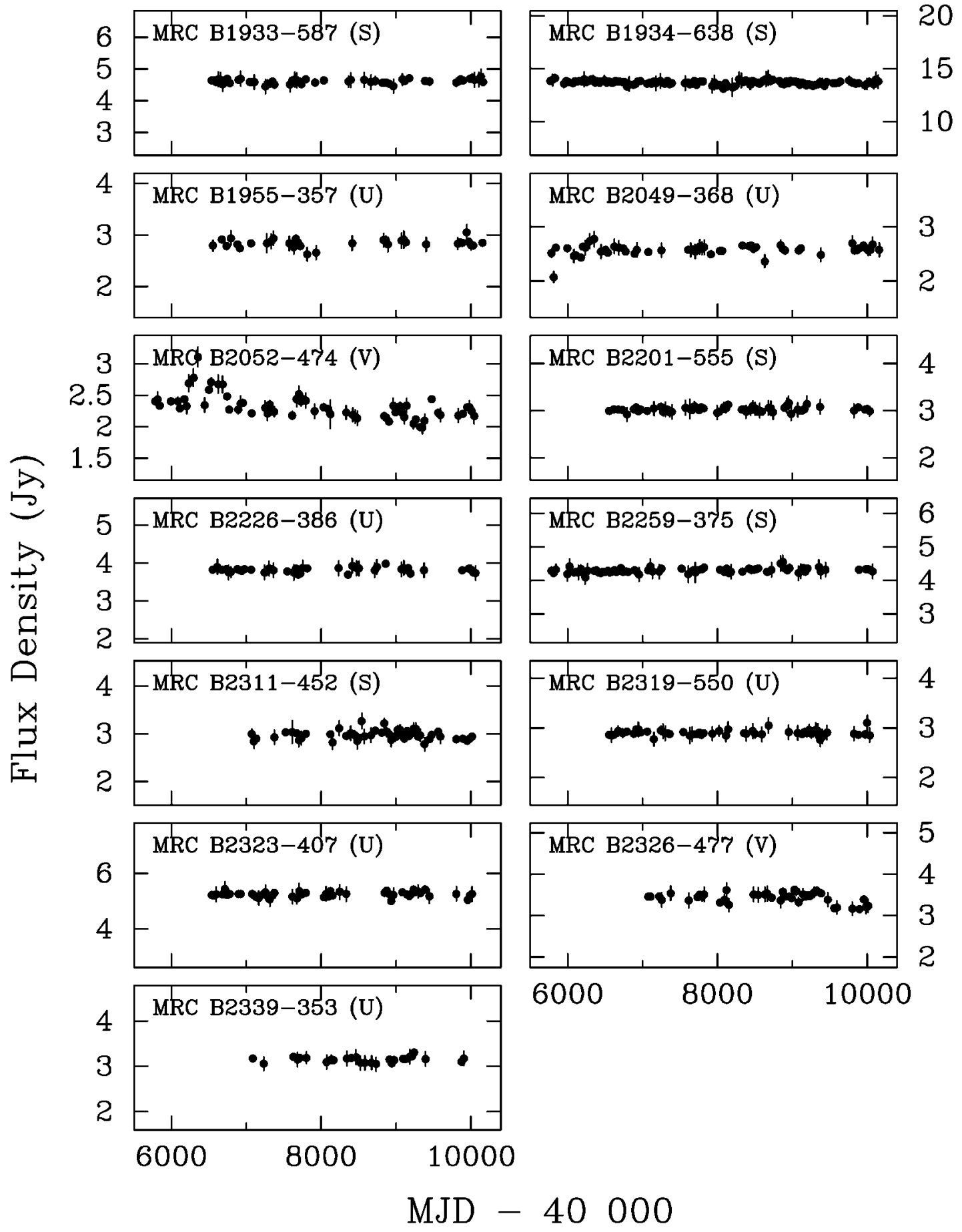

Figure 2-(continued)

MRC B1718-649: The nearest GHz-peaked-spectrum source, with a radio spectrum peaking near 3 GHz. VLBI imaging shows two sub-parsec-scale components separated by $\sim 2 \mathrm{pc}$ (Tingay et al. 1997).

MRC B1740-517: Crowded optical field; galaxy ID by di Serego Alighieri et al. (1994) is confirmed by an AAT CCD image (Burgess 1998).

MRC B1827-360: Compact ultra-steep-spectrum source identified with a galaxy in a very crowded field.
MRC B1829-718: Candidate source for defining the VLBI astrometric reference frame (Ma et al. 1998).

MRC B1854-663: Compact steep-spectrum source identified with a faint galaxy (Burgess 1998).

MRC B1921-293: See Paper I.

MRC B2052-474: Radio spectrum steep at low frequency, but flattens at high frequency; core dominated at $5 \mathrm{GHz}$, possibly triple (Burgess 1998). Detected as an X-ray source by the ROSAT All-Sky Survey (Brinkmann, Siebert \& Boller 1994). 


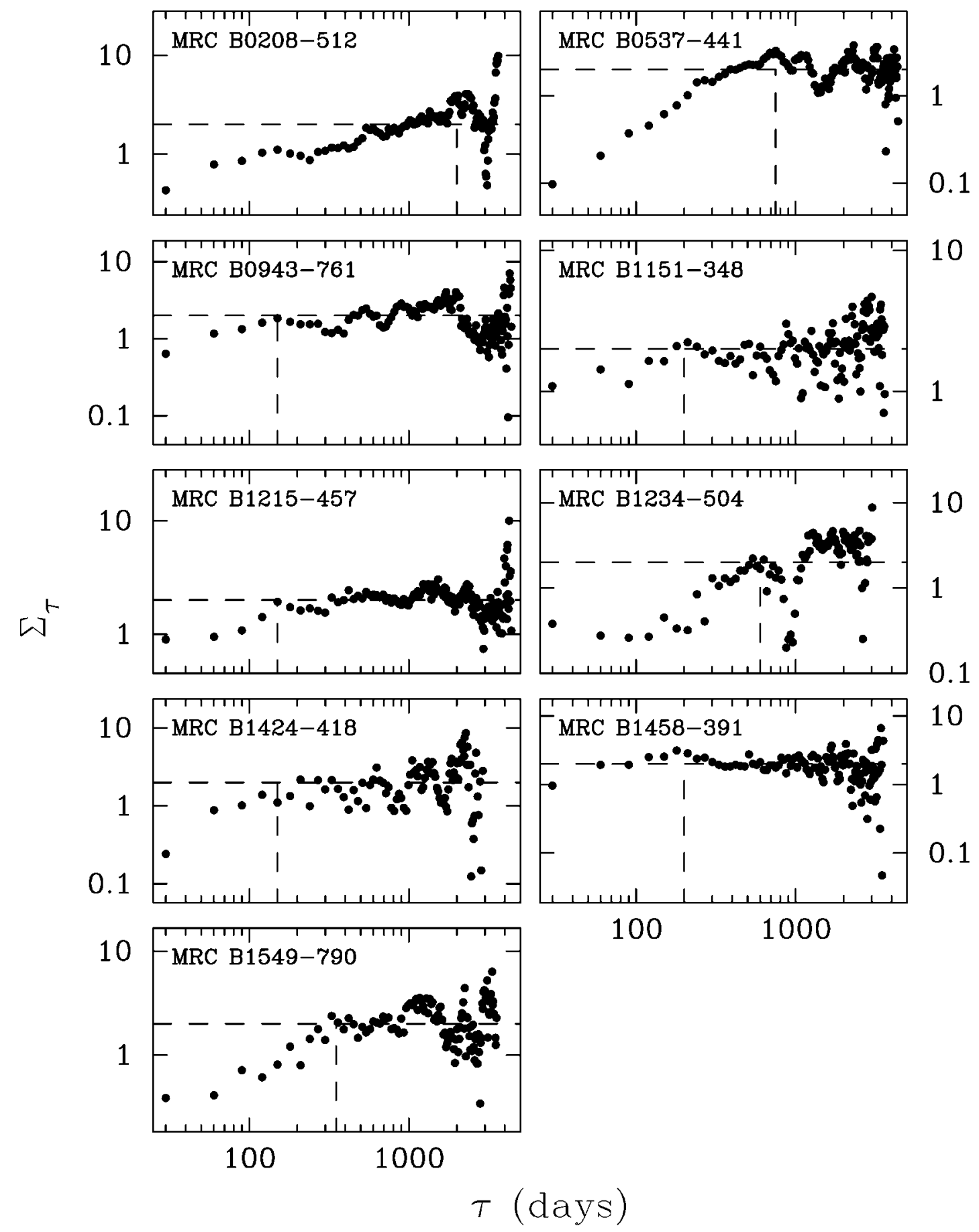

Figure 3-Structure functions for the 18 variable sources. The dashed horizontal line corresponds to $\Sigma_{\tau}=2$, the value at which a pure sinusoid will saturate. The dashed vertical line corresponds to the approximate lag at which the structure function saturates (the time scale for variability $\tau_{V}$ is defined to be twice this value).

MRC B2326-477: Detected as an X-ray source in the ROSAT All-Sky Survey (Brinkmann, Siebert \& Boller 1994). One of the set of defining sources for the VLBI astrometric reference frame (Ma et al. 1998).

\subsection{General Properties}

If the observed variability is a result of refractive scintillation in the Galactic interstellar medium (ISM), then we expect some sort of dependence of one of the modulation index $m=\sigma / \bar{S}$, characteristic timescale $\tau_{V}$ or their product, $m \tau_{V}$, on the Galactic latitude $b$ (e.g. Spangler et al. 1989; Ghosh \& Rao 1992). However, apart from a weak tendency for larger $\tau_{V}$ to occur at lower $|b|$, there is no obvious correlation in our data. This is not surprising given the large uncertainties in $\tau_{V}$ arising from the irregular sampling of the light curves, and the fact that there are few variable sources at high latitudes (14 of the 18 variable sources have $10^{\circ}<|b|<30^{\circ}$ ). 


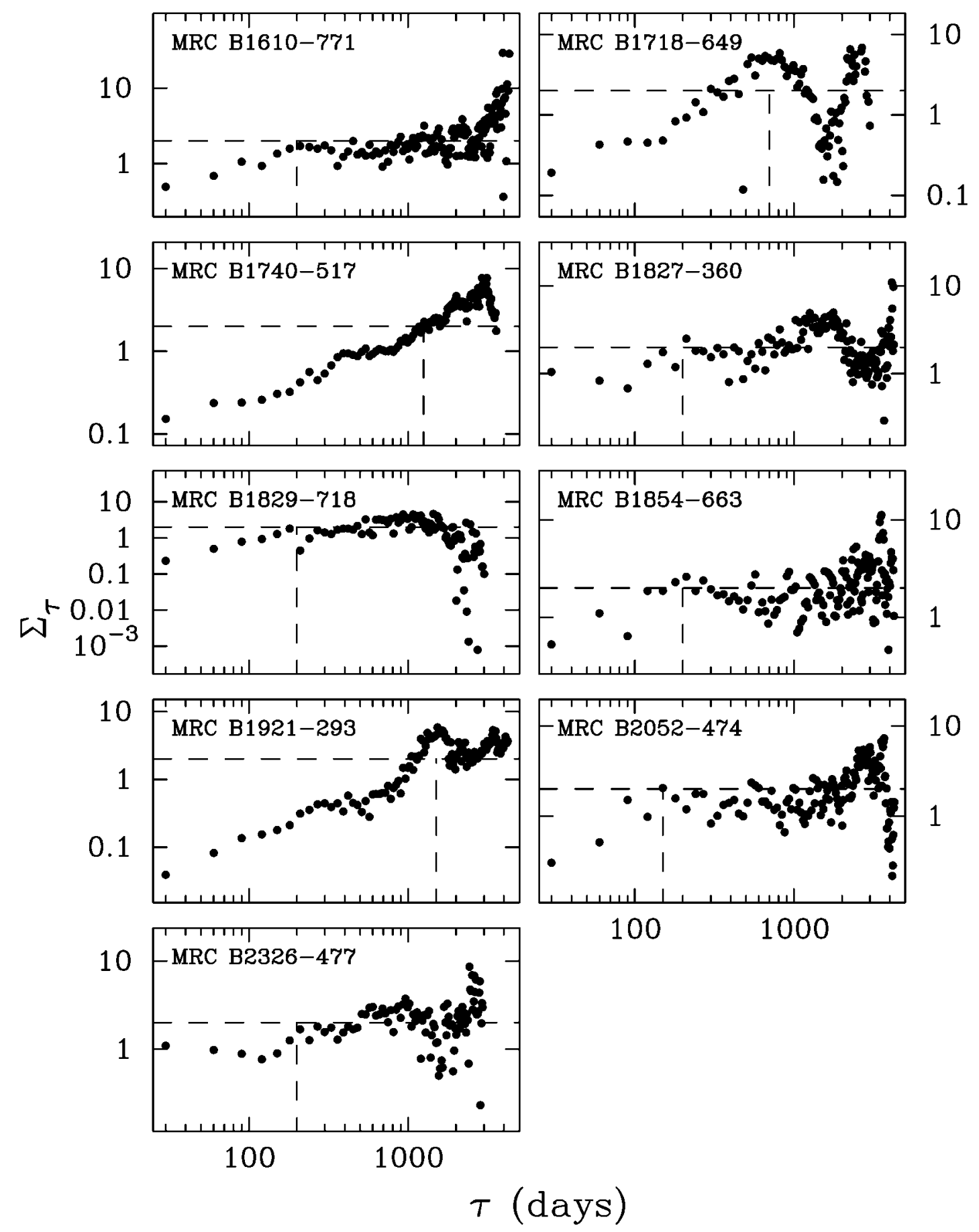

Figure 3-(continued)

An alternative indicator of the effects of the Galactic ISM is to test whether variable sources are more likely to be found at low latitudes. We consider this possibility in Figure 4, where we plot the ratio of variable sources $\left(N_{V}\right)$ to variable plus steady sources $\left(N_{V}+N_{S}\right)$ in different latitude bins. While the statistics are poor, there is a clear indication that sources are more likely to be variable at low latitudes, as found for northern hemisphere sources (Cawthorne \& Rickett 1985; Gregorini, Ficarra \& Padrielli 1986). This is unlikely to be caused by selection effects associated with a dependence of spectral index on Galactic latitude (cf. Cawthorne \& Rickett 1985), since the main criterion for source selection was angular size $\left(\theta<10^{\prime \prime}\right)$. Thus, the extensive monitoring data for the MOST calibrators provide good evidence that the variability observed at $843 \mathrm{MHz}$ arises from scintillation in the local ISM.

While spectral index was not considered in selecting the majority of the sample, Table 1 shows that two-thirds of the variables have flat or inverted 
Table 1. Properties of variable sources in our sample

\begin{tabular}{cccccccc}
\hline Source & $\begin{array}{c}b \\
(\mathrm{deg})\end{array}$ & $\begin{array}{c}\tau_{V} \\
(\text { days })\end{array}$ & $m^{\mathrm{a}}$ & Ident $^{\mathrm{b}}$ & $z$ & $\alpha^{c}$ & $\begin{array}{c}\text { LAS }^{\mathrm{d}} \\
\left({ }^{\prime \prime}\right)\end{array}$ \\
\hline MRC B0208-512 & $-61 \cdot 8$ & 2000 & $0 \cdot 047$ & $\mathrm{Q}$ & $1 \cdot 003$ & $-0 \cdot 23$ & $6 \cdot 0$ \\
MRC B0537-441 & $-31 \cdot 1$ & 1500 & $0 \cdot 139$ & $\mathrm{Q}$ & $0 \cdot 894$ & $+0 \cdot 25$ & $\ldots$ \\
MRC B0943-761 & $-17 \cdot 4$ & 300 & $0 \cdot 023$ & $\mathrm{~g}$ & $\ldots$ & $-0 \cdot 79$ & $2 \cdot 8$ \\
MRC B1151-348 & $+26 \cdot 3$ & 400 & $0 \cdot 019$ & $\mathrm{Q}$ & $0 \cdot 258$ & $-0 \cdot 49$ & $<2 \cdot 9$ \\
MRC B1215-457 & $+16 \cdot 5$ & 300 & $0 \cdot 019$ & $\mathrm{Q}$ & $0 \cdot 529$ & $-0 \cdot 59$ & $<1 \cdot 9$ \\
MRC B1234-504 & $+12 \cdot 0$ & 1200 & $0 \cdot 060$ & $\mathrm{Q} ?$ & $\ldots$ & $-0 \cdot 82$ & $<1$ \\
MRC B1424-418 & $+17 \cdot 3$ & 300 & $0 \cdot 074$ & $\mathrm{Q}$ & $1 \cdot 52$ & $-0 \cdot 47$ & $<2 \cdot 3$ \\
MRC B1458-391 & $+17 \cdot 0$ & 400 & $0 \cdot 022$ & $\mathrm{~g}$ & $\ldots$ & $-0 \cdot 67$ & $<2 \cdot 4$ \\
MRC B1549-790 & $-19 \cdot 5$ & 700 & $0 \cdot 050$ & $\mathrm{~g}$ & $0 \cdot 15$ & $-0 \cdot 29$ & $<1 \cdot 0$ \\
MRC B1610-771 & $-18 \cdot 9$ & 400 & $0 \cdot 052$ & $\mathrm{Q}$ & $1 \cdot 71$ & $-0 \cdot 13$ & $<1$ \\
MRC B1718-649 & $-15 \cdot 8$ & 1400 & $0 \cdot 060$ & $\mathrm{~g}$ & $0 \cdot 013$ & $+0 \cdot 21$ & $\ldots$ \\
MRC B1740-517 & $-11 \cdot 5$ & 2500 & $0 \cdot 063$ & $\mathrm{~g}$ & $\ldots$ & $-0 \cdot 08$ & $<1$ \\
MRC B1827-360 & $-11 \cdot 8$ & 400 & $0 \cdot 016$ & $\mathrm{~g}$ & $\ldots$ & $-1 \cdot 12$ & $<1 \cdot 5$ \\
MRC B1829-718 & $-24 \cdot 5$ & 400 & $0 \cdot 048$ & $\mathrm{~g}$ & $\ldots$ & $-0 \cdot 35$ & $\ldots$ \\
MRC B1854-663 & $-25 \cdot 5$ & 400 & $0 \cdot 022$ & $\mathrm{~g}$ & $\ldots$ & $-0 \cdot 86$ & $<1 \cdot 0$ \\
MRC B1921-293 & $-19 \cdot 6$ & 3000 & $0 \cdot 191$ & $\mathrm{Q}$ & $0 \cdot 352$ & $+0 \cdot 38$ & $\ldots$ \\
MRC B2052-474 & $-40 \cdot 4$ & 300 & $0 \cdot 084$ & $\mathrm{Q}$ & $1 \cdot 489$ & $-0 \cdot 34$ & $3 \cdot 9$ \\
MRC B2326-477 & $-64 \cdot 1$ & 400 & $0 \cdot 037$ & $\mathrm{Q}$ & $1 \cdot 489$ & $-0 \cdot 15$ & $\ldots$ \\
\hline
\end{tabular}

${ }^{a}$ Modulation index, defined by $m=\sigma / \bar{S}$.

${ }^{\mathrm{b}} \mathrm{Q}=$ quasar; $\mathrm{g}=$ galaxy.

c Spectral index $\alpha$ (where $S \propto \nu^{\alpha}$ ) between 408 and $2700 \mathrm{MHz}$ (or $4850 \mathrm{MHz}$ if $2700 \mathrm{MHz}$ flux density not available).

d Largest angular size at $5 \mathrm{GHz}$, measured with the Australia Telescope Compact Array (Burgess 1998).

spectra $(\alpha>-0 \cdot 5)$, consistent with source angular size being the main determinant of source variability. Surprisingly, the remaining third of the variables fall in the class of compact steep-spectrum (CSS) sources which are generally believed to be young sources still contained within their host galaxies, and not known to vary at high frequencies. The latter sources display a lower level of variability, as measured by the modulation index $m$, and in four of the six cases their $\mathrm{V}$ classification appears to be due to one-off events lasting $\sim 1$ year.

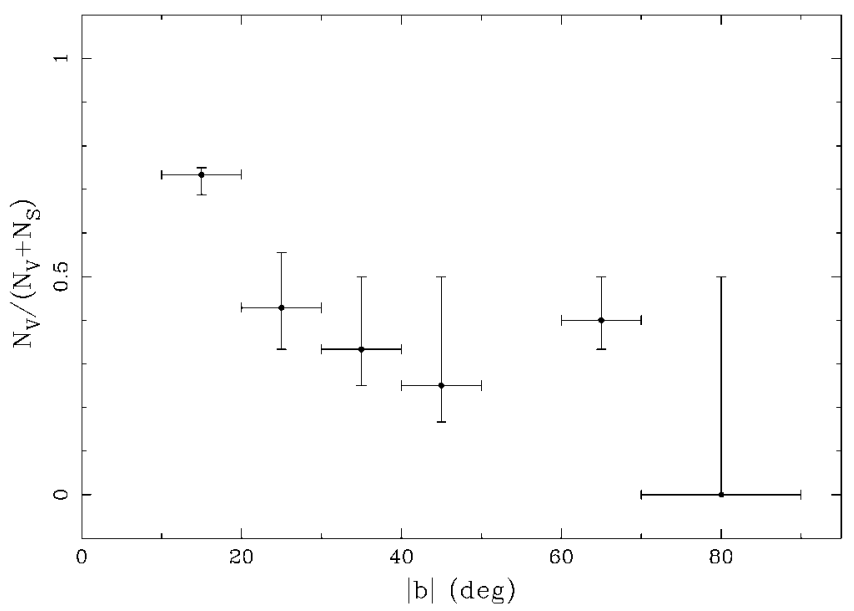

Figure 4-The fraction of variable sources as a function of Galactic latitude. Horizontal error bars represent the width of each latitude bin, while vertical error bars have been derived by computing the fraction of variable sources which results when half the undetermined sources in that bin are reclassified as either variable (upper limits) or steady (lower limits).
To investigate the variability properties of the MOST calibrator sample as a whole, in Figure 5 we have plotted $m$ versus $\alpha$ for all 55 sources. This figure shows a clear trend towards higher average modulation index as the radio spectrum flattens, with a suggestion of an upper envelope. Perhaps the simplest explanation for this behaviour in the unified model for powerful extragalactic radio sources is to link $m$ and $\alpha$ through the orientation of the radio axis to the line of sight (e.g. Orr \& Browne 1982). We assume that the 'core' of a classical triple source is the only part with components small enough in angular size to scintillate. If the core contribution dominates, as a consequence of Doppler boosting in the flat spectrum sources, even small fractional variations will be readily detected. However, the same fractional variations in the core of a steep-spectrum, lobe-dominated source will go undetected. We can therefore understand the trends in Figure 5 in a qualitative sense, and it is possible that a more detailed analysis may provide useful constraints on radio source models.

\section{Conclusions}

The 55 sources used for calibration purposes by the MOST at a frequency of $843 \mathrm{MHz}$ have been observed irregularly over a 13-year interval. We have developed an algorithm to process these data and produce a light curve for each source. Our analysis shows that 18 of these sources can be considered variable. There is some suggestion that these sources are distributed at lower Galactic latitudes than the 19 sources whose flux densities are 
unvarying. This suggests that variability at $843 \mathrm{MHz}$ on time scales of 1-10 years is predominantly due to scintillation in the Galactic ISM rather than effects intrinsic to the source. A possible correlation between modulation index and spectral index can be explained qualitatively in terms of a variation in the core fraction with orientation of the radio axis to the line of sight.

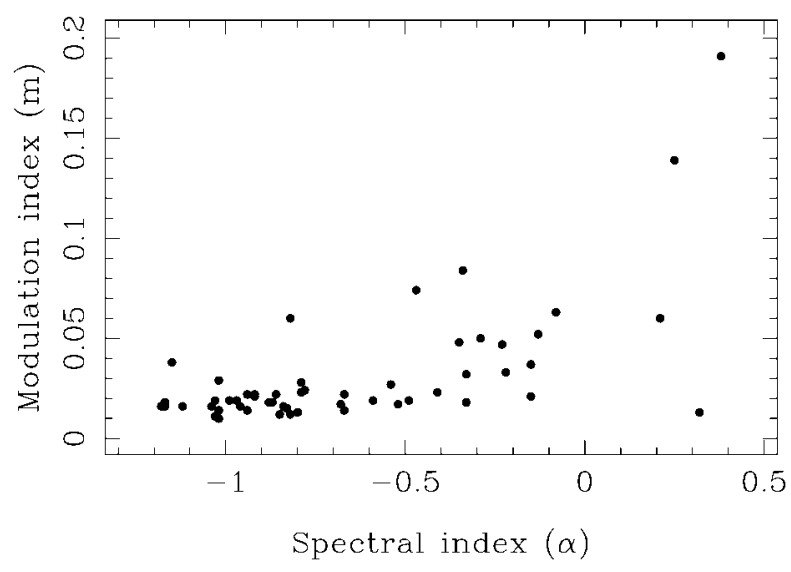

Figure 5-Plot of modulation index $m$ versus spectral index $\alpha$ for all 55 MOST calibrator sources. Note the tendency for $m$ to increase as the spectrum flattens.

\section{Acknowledgments}

We thank Ann Burgess for providing us with unpublished ATCA images of several sources and for supplying us with her improved meridian-distance gain curve. We also thank Duncan CampbellWilson, Lawrence Cram, Jean-Pierre Macquart, Gordon Robertson, Mark Walker and Taisheng Ye for useful discussions and advice, and an anonymous referee for a careful reading of the manuscript. This research has made use of the NASA/IPAC Extragalactic Database (NED), operated by JPL under contract with NASA. The MOST is supported by grants from the Australian Research Council, the University of Sydney Research Grants Committee, and the Science Foundation for Physics within the University of Sydney. BMG acknowledges the support of an Australian Postgraduate Award and of NASA through Hubble Fellowship grant HF-01107·01-98A awarded by the Space Telescope Science Institute, which is operated by the Association of Universities for Research in Astronomy, Inc., for NASA under contract NAS 5-26555.

\section{References}

Bertsch, D. L., et al. 1993, ApJ, 405, 21

Brinkmann, W., Siebert, J., \& Boller, T. 1994, A\&A, 281, 355

Burgess, A. M. 1998, PhD thesis, University of Sydney

Campbell-Wilson, D., \& Hunstead, R. W. 1994, PASA, 11, 33 (Paper I)

Cawthorne, T. V., \& Rickett, B. J. 1985, Nature, 315, 40

di Serego Alighieri, S., Danziger, I. J., Morganti, R., \& Tadhunter, C. N. 1994, MNRAS, 269, 998

Ghosh, T., \& Rao, A. P. 1992, A\&A, 264, 203

Gregorini, L., Ficarra, A., \& Padrielli, L. 1986, A\&A, 168, 25

Hughes, P. A., Aller, H. D., \& Aller, M. F. 1992, ApJ, 396, 469

Hunstead, R. W. 1972, ApJ Lett., 12, 193

Hunstead, R. W. 1991, Aust. J. Phys., 44, 743

Hunstead, R. W., \& Murdoch, H. S. 1980, MNRAS, 192, $31 \mathrm{P}$

Kaspi, V. M., \& Stinebring, D. R. 1992, ApJ, 392, 530

Kedziora-Chudczer, L., Jauncey, D. L., Wieringa, M. H., Walker, M. A., Nicolson, G. D., Reynolds, J. E., \& Tzioumis, A. K. 1997, ApJ, 490, L9

Kesteven, M. J. L., Bridle, A. H., \& Brandie, G. W. 1976, AJ, 81, 919

King, E. A., et al. 1993, in Sub-arcsecond Radio Astronomy, ed. R. J. Davis \& R. S. Booth (Cambridge Univ. Press), p. 152

Large, M. I., Campbell-Wilson, D., Cram, L. E., Davison, R. G., \& Robertson, J. G. 1994, PASA, 11, 44

Large, M. I., Mills, B. Y., Little, A. G., Crawford, D. F., \& Sutton, J. M. 1981, MNRAS, 194, $693^{4}$

Ma, C., et al. 1998, AJ, 116, 516

Mills, B. Y. 1981, PASA, 4, 156

Mitchell, K. J., Dennison, B., Condon, J. J., Altschuler, D. R., Payne, H. E., O'Dell, S. L., \& Broderick, J. J. 1994, ApJS, 93, 441

Murphy, D. W., et al. 1993, in Sub-arcsecond Radio Astronomy, ed. R. J. Davis \& R. S. Booth (Cambridge Univ. Press), p. 243

Orr, M. J. L., \& Browne, I. W. A. 1982, MNRAS, 200, 1067

Preston, R. A., et al. 1989, AJ, 98, 1

Qian, S. J., Britzen, S., Witzel, A., Krichbaum, T. P., Wegner, R., \& Waltman, E. 1995, A\&A, 295, 47

Rickett, B. J., Coles, W. A., \& Bourgois, G. 1984, A\&A, 134,390

Robertson, J. G. 1991, Aust. J. Phys., 44, 729

Shapirovskaya, N. Y. 1978, Sov. Astron., 22, 544

Spangler, S., Fanti, R., Gregorini, L., \& Padrielli, L. 1989, A\&A, 209, 315

Tingay, S. J., et al. 1996, ApJ, 464, 170

Tingay, S. J., et al. 1997, AJ, 113, 2025

Tzioumis, A. K. 1987, PhD thesis, University of Sydney 